\title{
Aproximación al mundo gráfico-sonoro de domingo lobato: Transcripción y análisis de sus siete Bagatelas para piano
}

\section{An approach to the graphic-sonorous world of domingo lobato: Analysis and transcription of his seven Bagatelas for piano}

\author{
María Eugenia Cosio Hernández ${ }^{a}$
}

\begin{abstract}
:
Domingo Lobato was a prolific but little known composer whose works have not had enough exposure. Thus, it is intended to show a little more of his compositive works by the exemplified exposition of his unpublished work Bagatelas. The main objectives are based on the transcription, analysis and the study of the manuscripts, for posterity it is hoped that the works will be properly interpreted and given proper exposure.

The intricate stylistic treatment shows this work required an eclectic study methodology. It was used apart from the formal analysis the gregorian theory, modal harmony, quartal harmony and post-Wagnerian harmony.

One of the biggest contributions of Lobato was the use of the compositional orthodoxy in a vanguardist way, using the aesthetic and compositive classical canons to create a new and particular sound language.

The present work represents the first approach to Lobato's work from a novel analytical perspective for study, transcriptional and difussional purposes. It is worth mentioning that both Lobato's work and his disciples and contemporaries have been rarely referred to by current musicology.
\end{abstract}

\section{Keywords:}

Lobato, Bagatelas, transcription, philological analysis.

\section{Resumen:}

Domingo Lobato fue un prolífico, pero poco conocido compositor cuyas obras no han tenido difusión suficiente. Por ello, se pretende dar a conocer un poco más de su acervo compositivo mediante la exposición ejemplificada de su obra inédita Bagatelas. Los objetivos principales se cifran en la transcripción, estudio y análisis de los manuscritos, para con posterioridad ser susceptibles de ser interpretados y así darles la pertinente difusión.

El intrincado tratamiento estilístico que manifiesta la obra requirió de una metodología de estudio ecléctica. Para ello se recurrió aparte del análisis formal a la teoría gregoriana, armonía modal, armonía cuartal y armonía post-wagneriana.

Uno de los mayores aportes de Lobato radicó en la utilización de la ortodoxia compositiva de manera vanguardista, empleando los cánones estéticos y compositivos clásicos para crear un lenguaje sonoro novedoso y particular.

El presente trabajo representa el primer acercamiento a la obra de Lobato, desde una perspectiva analítica novedosa con fines de estudio, trascripción y difusión. Cabe mencionar que, tanto la obra de Lobato, como la de sus discípulos y coetáneos ha sido escasamente referida por la musicología actual.

\section{Palabras Clave:}

Lobato, bagatelas, transcripción, análisis filológico

\section{Introducción}

Hablar de los referentes musicales en el México del siglo

XX es mencionar entre otros a Carlos Chávez (1899-1978),

Silvestre Revueltas (1899-1940), Miguel Bernal Jiménez

${ }^{a}$ Autor de Correspondencia, https://orcid.org/0000-0001-9283-9812,Email: maru_cosio@live.com.mx 
(1910-1956), Blas Galindo (1910-1943) o José Pablo

Moncayo (1912-1958). Sin embargo, eminentes

compositores como Domingo Lobato (1920-2012),

Guillermo Pinto Reyes (1921-1997) o Paulino Paredes

(1913-1957) son poco conocidos a pesar de su vasto legado compositivo. Posiblemente porque al no radicar en la capital del país donde los movimientos culturales tenían su epicentro legitimado por el Estado, derivó en una modesta difusión de sus obras, siempre ligadas al espectro de lo local.

El presente trabajo pretende dar a conocer un poco más el acervo compositivo de Lobato mediante la exposición ejemplificada del proceso de transcripción de su obra inédita Bagatelas. * Los principales objetivos se cifran en la transcripción, estudio y análisis de los manuscritos, para con posterioridad ser susceptibles de ser interpretados y así darles la pertinente difusión.

Para el objetivo específico de la transcripción se utilizaron las herramientas contenidas en el texto de Chailley (1950), como el uso de aplicaciones de edición de partituras y el análisis musical. El programa computacional Finale fue utilizado para dicho cometido.

En palabras de Lester (2005) “...la melodía y la armonía las generan relaciones motívicas entre grupos de alturas. El análisis de esta música implica la localización de esos motivos y la comprensión de cómo se los emplea." Por ello, para el análisis rítmico y melódico se recurrió al análisis de la estructura motívica, al tratamiento de los intervalos y alturas, a los motivos rítmicos, la textura y a la frase y forma que plantea en su texto Enfoques analíticos de la música del siglo XX.

\section{Breve esbozo biográfico de Domingo Lobato}

\footnotetext{
* Obra para piano compuesta en 1982. Integrada por las siguientes partes: 1. Ajijic, 2. (Sin título), 3. Chapala, 4. Agua Azul, 5. Expiatorio, 6. (Sin título) y 7. (Sin título) $\dagger$ Fundador y director de la Escuela Superior de Música Sagrada de la Arquidiócesis de Michoacán de 1914 a 1961.

* (1881-1972). Discípulo de Francisco Navarro, José Franco y Juan B. Paulín. Enseñó piano y solfeo en la Universidad de
}

El compositor y su creación no se sitúan en el tiempo como un elemento aislado sino influido por el contexto social, histórico, político y económico del periodo en el que vivió. Por ello se incluye a continuación un breve esbozo biográfico que amplía el panorama en el devenir del compositor en su época y, desde este posicionamiento, comprender de manera más eficiente su obra:

Originario de Morelia, Michoacán, nació el 4 de agosto de 1920. Desde los seis años formó parte del coro de niños del templo de San Juan Bautista, donde descubrió su afición por la música. Cursó sus estudios musicales en la Escuela Superior de Música Sacra de Morelia después de haber pasado por un proceso riguroso de selección y reclutamiento y gracias a la invitación del canónigo José María Villaseñor (1880-1961) quien fue el principal benefactor en su formación musical. ${ }^{\dagger}$

A los diez años, fue elegido por su voz y sentido musical para formar parte del Coro de Infantes de la Catedral de Morelia. Realizó estudios musicales en la Escuela Superior de Música Sagrada de Morelia bajo la dirección de maestros como Miguel Bernal Jiménez (19101956), con quien cursó las carreras de Canto Gregoriano, Composición y Órgano, y de Ignacio Mier Arriaga (18811972), ${ }^{*}$ de quien fue discípulo en la materia de piano.

En 1943 recibió el título de Maestro en Composición, donde tuvo por sinodal, entre otros, al compositor jalisciense José Rolón. En 1945 obtuvo el de Magisterio en Canto Gregoriano. Para el año de 1946 fue invitado a ocupar la cátedra de composición en la Escuela de Música Sacra de Guadalajara a petición expresa de su director, Manuel de Jesús Aréchiga. ${ }^{\S}$ En 1951 colaboró en la Escuela de Bellas Artes del Estado y en 1952 fue requerido para formar parte del cuerpo docente de la Escuela de Música de la Universidad de Guadalajara. En

San Nicolás de Hidalgo. Fundó la Escuela de Bellas Artes y fue maestro de piano de la Escuela Superior Diocesana de Música Sagrada de Morelia.

$\S$ (1903-1984). Pianista, organista, director de coro y orquesta y pedagogo. Egresado del Pontificio Instituto de Música de Roma. Primer director de la Escuela Superior Diocesana de Música Sagrada de Guadalajara. 
dicha institución impartió las cátedras de Armonía, Composición y Análisis Musical hasta el año de su jubilación 1973.

En 1956 asumió el puesto como director de la Escuela de Música de la Universidad de Guadalajara y fungió como director de la Orquesta Sinfónica de Guadalajara (1956-1974). Gracias a su desempeño la escuela creció en número de alumnos. También diseñó los planes de estudio y así conformó las carreras de Instructor de Música y Ejecutante Profesional de Instrumentos, así como Profesor de Canto. Desempeñó el cargo durante dieciocho años.

Entre otras actividades, durante esa época organizó conciertos juveniles y temporadas de ópera en un acto. La escuela ofrecía una atractiva imagen a la ciudadanía por las constantes actividades que ofrecía.

En 1968 el Ballet Folclórico de la Universidad de Guadalajara fue invitado a participar en las festividades programadas en la Ciudad de México con motivo de las Olimpiadas de ese año. Siendo Lobato responsable del coro y por su brillante participación, recibió una medallareconocimiento del entonces presidente de la República, Gustavo Díaz Ordaz (1911-1979).

En 1969 tuvo la oportunidad de viajar a la entonces República Federal de Alemania donde dictó conferencias acerca de la música mexicana y tomó nota de las tendencias de vanguardia y programas de estudio en la educación musical. Dicha información le sirvió para reorientar los planes de estudio de la Escuela de Música de la Universidad de Guadalajara.

En la Escuela Superior de Música Sacra de Guadalajara siguió impartiendo sus cátedras para la licenciatura en Canto Gregoriano y la licenciatura en Composición desde 1946 hasta una semana antes de su muerte en 2012.

A lo largo de su vida fue objeto de numerosos reconocimientos y premios por su destacada labor musical.

\footnotetext{
* Obra para piano compuesta en 1945. Conformada por las siguientes partes: Danzinela, Serenata, El Amor al Trompo Rojo, Habanera y Mamá Osa e Hijo.
}

Su creación musical abarca música religiosa, música para órgano, conciertos, música escénica, música coral, música de cámara, cuartetos para cuerdas, tríos, ensambles de cámara, así como una vasta producción de música para piano. Siguiendo a Cosio (2018, p. 36) es posible ver la dimensión musical del compositor michoacano:

Lobato fue un músico muy completo e innovador de la música y de la cultura musical mexicanas. Transitó por diversos estilos compositivos como el romanticismo e impresionismo hasta lenguajes sonoros de vanguardia; trayecto creativo que impregnó a varias de sus obras con elementos y facturas características del impresionismo, serialismo, dodecafonismo, entre otros.

Su legado tiene resonancia en la música actual mexicana. Gracias a su visión vanguardista la enseñanza de la música en Guadalajara fue reformada. Entre sus discípulos se encuentran los destacados músicos Hermilio Hernández (1931-2008), Víctor Manuel Amaral (1933-2019), José Guadalupe Flores (1947-), Francisco Javier Hernández (1937-), Leonor Montijo (1932-2018), Julieta Marrón (1960-) y Ana Eugenia González Gallo (1938).

\section{Antecedentes}

La autora del presente trabajo tuvo el privilegio de tener al maestro como profesor de Canto Gregoriano y del taller de Composición en la Escuela Superior de Música Sacra de Guadalajara (del año 2008 al 2009). Allí se suscitó el primer acercamiento con su obra pianística al interpretar la Suite de los Niños. * Con dicha obra se propició una exploración de un lenguaje compositivo, ignoto hasta el momento para la autora de este texto. En primera instancia, es posible reconocer las dificultades tanto técnicas como musicales que supone la ejecución de la obra. Lo anterior deriva de un estilo compositivo poco abordado en las escuelas de música y que se aparta, en cierta medida, del canon pedagógico usual del piano. Esas dificultades fueron resueltas con la inestimable y generosa ayuda del propio maestro, lo que le permitió establecer una fluida relación con él. Posteriormente, el tema de tesis de la autora para la 
obtención del grado de Maestría en Educación y Expresión para las Artes en la Universidad de Guadalajara, fue el análisis y transcripción de las dos obras inéditas para piano del maestro Lobato Para Sophia (1992) y Tres Danzas (1994) y cuyo resultado se puede encontrar en la tesis Generación de un producto de creación: Obra del maestro Domingo Lobato: Para Sophia y Tres Danzas (2018).

La autora recibió por parte del albacea del acervo compositivo de Lobato, su hijo el ingeniero José Lobato, la encomienda de transcribir el manuscrito de una de sus obras inéditas titulada Bagatelas para su posterior interpretación y difusión. El resultado de dicho encargo es el tema de la presente comunicación.

De acuerdo con la clasificación de Gabriel Pareyón los títulos de sus obras editadas para piano son los siguientes: * Suite número 1 (1945), Seis danzas (1956), Sonata número 1 (1957), Sonatina (1960), Sonata número 2 (1961), Cuatro estudios breves atonales (1975), Once estudios elementales (1988), Sonata número 3 (1988), Tres preludios (1999). Sin embargo, existe un número importante de obras que se conservan aun en manuscrito, que, si bien no están reconocidas oficialmente dentro del catálogo de su producción musical, constituyen una parte sustancial de su creación pianística.

\section{Desarrollo del trabajo}

El espectro estilístico de Lobato comprende acercamientos con procedimientos usuales en distintos periodos musicales como: el romanticismo, el impresionismo, el atonalismo, el dodecafonismo y el serialismo. Por tanto, para la comprensión cabal de la obra fue necesario la realización de un amplio análisis filológico en donde se desarrollan los elementos constitutivos que se exponen a continuación.

\footnotetext{
* Compositor polimático y musicólogo mexicano. Su música combina aspectos más abarcadores de la lingüística y el habla humana, modelos matemáticos y modelos que provienen de la vocalización de las aves. Tutor del Programa de Posgrado de la Escuela Nacional de Música de la UNAM. Miembro titular del CENIDIM-INBA donde
}

\section{Análisis}

En palabras de Nagore (2004) "Es difícil prescindir de la representación gráfica en el trabajo analítico por lo que no debemos perder de vista el valor relativo y simbólico de la notación o la representación gráfica". Por ello, para facilitar la lectura del manuscrito se realizó un sistema comparativo que permitió encontrar las figuras principales plenamente identificadas de la notación musical dentro de la escritura del maestro para que sirvieran como guía en las posteriores comparaciones y, posteriormente, corroborar con el símbolo estándar de la escritura de los signos musicales. Además se procedió a familiarizarse con todo el estilo de escritura de Lobato para poder inferir con mayor precisión las grafías correctas de cada sección.

Desde la perspectiva de la autora de este texto, se considera que una de las mayores dificultades de la música de Lobato deriva de la utilización de múltiples recursos estilísticos. La metodología del análisis de la factura musical estuvo basada en los aspectos rítmico, melódico, armónico y estructural; se utilizó la teoría gregoriana, armonía modal, armonía cuartal y armonía postwagneriana. El tratamiento estilístico de la obra evidencia la presencia de motivos cercanos a los utilizados por compositores impresionistas. En particular la bagatela 3, Chapala, presenta texturas que evocan la música de Debussy y Ravel.

Las Figuras 1 y 2 muestran uno de los retos que representó el descifrar la grafía-musical, pues el manuscrito presenta muchas tachaduras, enmendaduras, secciones borradas, figuras no claras, incluso había notas que abarcaban hasta tres espacios o tres líneas en el pentagrama, lo cual hizo muy difícil reconocer los signos correctos. Esa problemática se manifiesta a lo largo de todo

tiene a su cargo el Seminario de Ciencias y Teorías de la Música. Ha publicado libros y artículos especializados sobre filosofía estética, ecología, semiótica, e historia y teoría de la música. Es miembro del Sistema Nacional de Creadores de Arte. Actualmente coordina el Congreso Internacional de Música y Matemáticas. 
el manuscrito. Esas complicaciones no sólo se dieron en cuanto a la grafía de las notas sino también en la agógica. En general, la partitura manuscrita aunada al lenguaje musical hizo muy difícil comprender qué pretendió escribir en realidad el maestro en algunas secciones. Un objetivo importante fue transcribir el manuscrito denominado Bagatelas a un programa de edición de partituras, en este caso Finale, para facilitar su posterior conservación, ejecución y difusión.

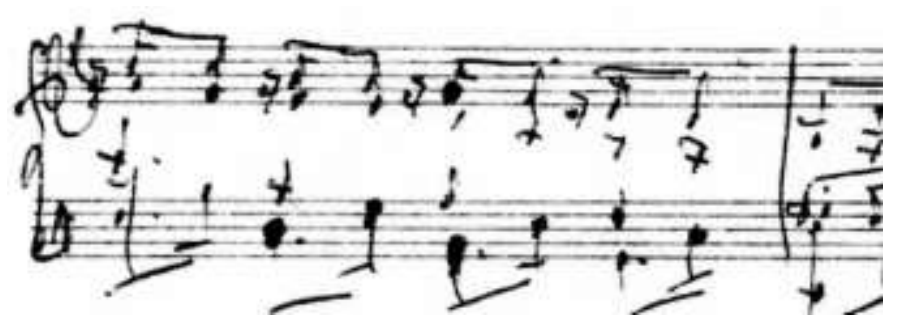

Figura 1. Sección de la Bagatela número uno Ajijic
Danzas (1965), donde el motivo rítmico presente a lo largo de la obra está conformado por una corchea y dos semicorcheas. Además, en el primer movimiento de su Sonata número dos (1961), que expone un patrón rítmico de corchea-negracorchea-negra en compás de 6/8 seguido de un cambio de compás a 4/8 integrado por dos negras a lo largo de toda la obra como se muestra a continuación:

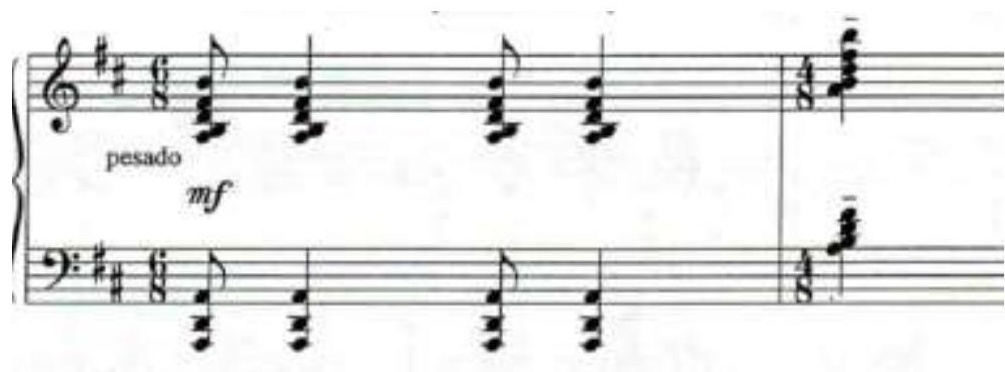

Figura 3. Fragmento de la Sonata para piano no. 2 de Domingo Lobato

Cada bagatela es una composición independiente, algunas de ellas tienen un marcado carácter rítmico, tal es el caso de la bagatela número uno Ajijic. Por otro lado, las bagatelas número dos (sin título) y número cinco Expiatorio, muestran una factura donde el compositor enfatizó el aspecto melódico. En la bagatela número dos, es posible observar que la frase musical se presenta en los primeros dos compases, manifiesta un ritmo muy simple y concede el protagonismo a la melodía, misma que tiene su réplica en los siguientes dos compases.

Se puede apreciar en el siguiente gráfico un motivo rítmico muy marcado que se repite a lo largo de la bagatela. Este recurso que consiste en la utilización de múltiples patrones rítmicos da a su obra un marchamo distintivo en la producción del compositor. Aparece en distintas obras como en Habanera de su Suite de los Niños (1945), donde el ritmo constante de la voz acompañante es de corchea con puntillo y semicorchea. Otro ejemplo de lo anterior se encuentra en su danza Vihuelas y Guitarrones de su compendio Seis 


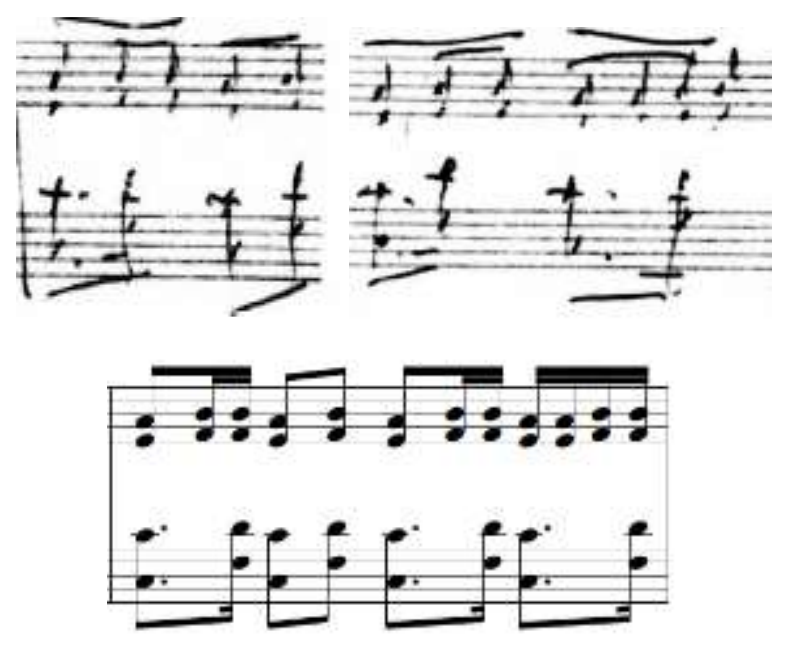

Figura 4. Fragmento de la bagatela número uno Ajijic
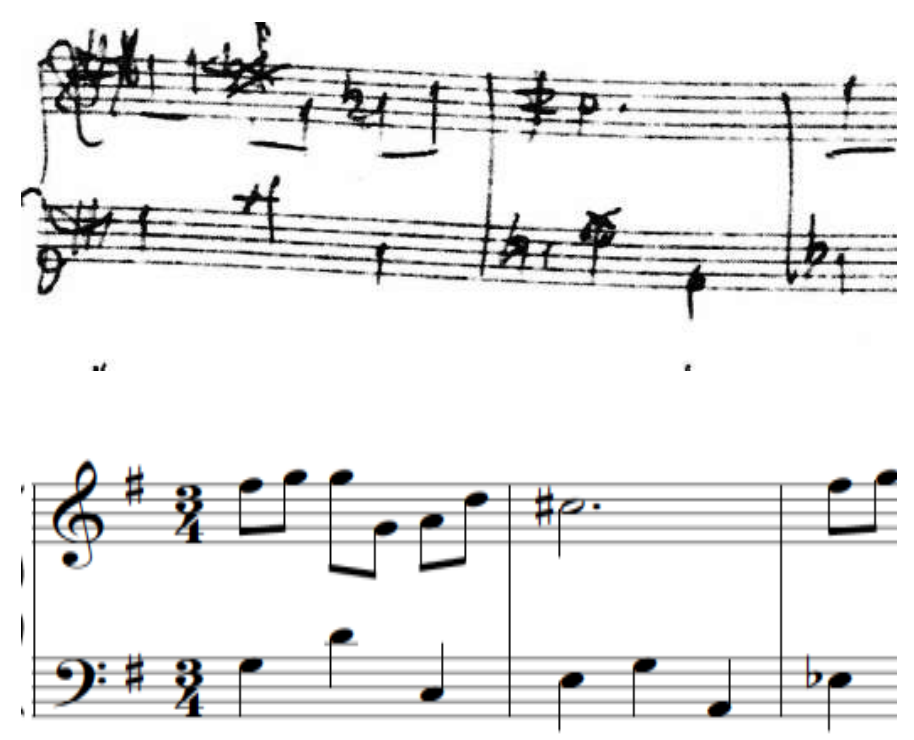

Figura 5. Fragmento de la bagatela número dos.

En este fragmento de la bagatela número cinco, titulada Expiatorio, se evidencia una melodía con reminiscencias modales gregorianas:

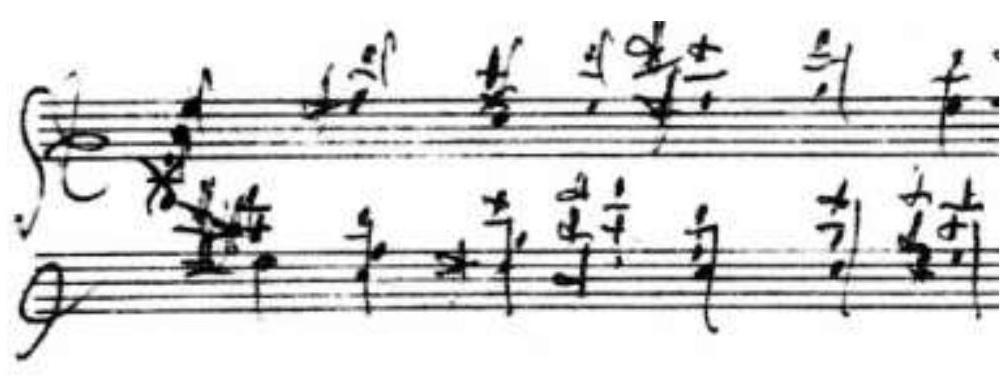

Figura 6. Fragmento de la bagatela número cinco

Expiatorio.

Incluso su título Expiatorio concuerda con el carácter eclesial de la obra, ya que hace alusión al templo Expiatorio de la ciudad de Guadalajara. ${ }^{*}$ Puede decirse que Lobato utilizó toda la ortodoxia compositiva de manera vanguardista, empleando los cánones estéticos y compositivos clásicos para crear un lenguaje sonoro novedoso y particular, lo cual significa uno de sus mayores aportes. Con esta evidencia se puede resaltar que, al igual que Prokofiev mencionó que aún había cosas bellas que decir en Do mayor, Lobato exploró los límites musicales a través de la síntesis de distintos estilos y facturas musicales para así crear un lenguaje personal sin precipitarse a adoptar estilos de moda que le resultaran ajenos.

En la bagatela número tres la figura rítmica predominante en la voz del acompañamiento es un seisillo. Dicha voz es conducida por intervalos melódicos de segundas y terceras menores. Puede afirmarse que la bagatela evoca en cierta manera el impresionismo de Ravel en sus Juegos del agua (1901) y de Debussy en sus Jardines bajo la lluvia (1903), donde precisamente es utilizado dicho patrón rítmico de seisillo en la voz acompañante, lo cual busca generar una sensación auditiva que recuerda el movimiento del agua.

después de la Catedral de Guadalajara. Considerado como una expresión del neogótico en México.
* Llamado originalmente Templo del Santísimo Sacramento, es considerado el segundo en importancia 

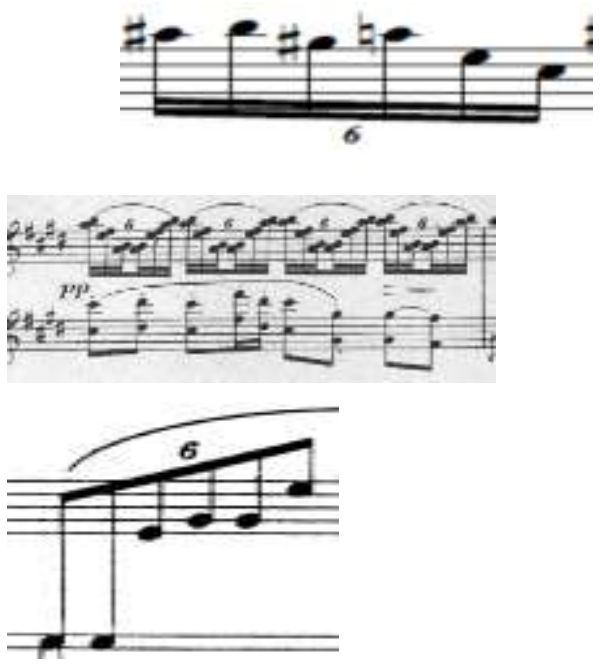

Figura 7. Motivo rítmico de seisillo en Chapala de Lobato,

Juegos del agua de Ravel y Jardines bajo la lluvia de

Debussy respectivamente.

En la bagatela número siete ( $\sin$ título) hay una variación de un fragmento de la canción Flores Negras lo que confirma la importancia que para Lobato tenía la utilización de elementos de melodías populares al incorporarlos en sus obras. *

\section{Conclusiones}

La realización del presente trabajo ha permitido a su autora profundizar un poco más en el lenguaje compositivo de Domingo Lobato. El estudio ha representado un gran reto técnico puesto que, en la música de Lobato y particularmente en estas bagatelas, la técnica pianística está en función de la música. No es que se adaptara a las limitantes técnicas del instrumento, en este caso del piano, sino que lo que el compositor buscaba decir con su música era más importante que la forma de ejecutarlo. Otro reto fue el análisis estilístico y de factura, sobre todo a la hora de reconocer la variedad de estilos que maneja Lobato, la forma en que los fusiona para crear su lenguaje propio. Ese aspecto fue muy enriquecedor para quien esto escribe, así como también lo fue el poder encontrar los estilos y la manera en que los apropió para dar paso a su estilo propio.

En resumen, además de haber realizado un esbozo biográfico para contextualizar al compositor, su circunstancia y su obra, se ha logrado satisfactoriamente la transcripción del manuscrito previo análisis melódico, rítmico y estilístico, lo que permite a la autora tener el convencimiento de haber realizado un trabajo meticuloso que interpreta fielmente la idea del compositor. Esto constituye un aliciente para seguir transcribiendo las obras inéditas de Lobato y así legar partituras que pasen no sólo a engrosar el catálogo de su producción pianística, sino a constituir un referente para las generaciones actuales y venideras tanto pedagógica como musicalmente hablando, puesto que quien esto escribe es consciente de la importancia que tuvo Domingo Lobato en el quehacer musical no sólo de Michoacán y Jalisco sino de todo México.

\section{Referencias}

\section{Bibliografía}

ALTAMiRANO, J. (1999). Biografía M. Ilustre Sr. Cngo. Dean D. José Ma.Villaseñor. Manuscrito no publicado.

CHAILLEY, J. (1950). Les notations musicales nouvelles. Paris: Alphonse Leduc.

COSIO, M. (2018). Generación de un producto de creación: Obra del maestro Domingo Lobato: Para Sophia y Tres Danzas. (Tesis de maestría) Universidad de Guadalajara. Jalisco, México.

LESTER, J. (2005). Enfoques analíticos de la música del siglo XX. Madrid, España: Akal.

\footnotetext{
* Bolero compuesto por el cubano Sergio de Karlo en la Ciudad de México en 1932 dedicado a una mujer michoacana.
} 
LOBATO, D. (1982). Siete Bagatelas para Piano. Manuscrito inédito: ADL

NAGORE, M. (2004). El análisis musical, entre el formalismo y la hermenéutica. Revista Músicas al Sur.

PAREYÓN, G. (2007). Diccionario enciclopédico de música en México. Jalisco, México: Universidad Panamericana.

\section{Recursos electrónicos}

CRUZ, G. (2018). Domingo Lobato Bañales. Lugar de publicación: Instituto de Investigaciones históricas, políticas,económicas y sociales. Recuperado de: https://institutohistorico.org/domingo-lobato-banales/

DE KARLO, S. (2020). Compositores y autores: Sergio de Karlo. E.U.: Tiempo de Boleros. Recuperado de : http://tiempodeboleros.eu/biografia/compositores/1314$\underline{\text { sergio-de-karlo }}$

El Templo Expiatorio de Guadalajara. (2018). El Templo Expiatorio de Guadalajara. México.: Entorno turístico. Recuperado de :

https://www.entornoturistico.com/el-templo-expiatorio-deguadalajara/

Pareyón, G. (2009). Página web. Posgrado Música. Recuperado de: https://www.posgrado.unam.mx/musica/div/docentes/perso $\underline{\text { nal_docente/pareyon.html\# }}$

Lobato Bañales, Juan Domingo. (2017). Lobato Bañales, Juan Domingo. México.: Enciclopedia histórica y biográfica de la Universidad De Guadalajara. Recuperado de: http://enciclopedia.udg.mx/biografias/lobato-banalesjuan-domingo

\section{Índice de ilustraciones}

1. Sección de la Bagatela número uno Ajijic.

2. Comparación de Claves de sol en la escritura de Lobato y en su versión estándar.
3. Fragmento de la Sonata para piano no. 2

4. Fragmento de la Bagatela número uno Ajijic

5. Fragmento de la bagatela no. 2.

6. Fragmento de la bagatela número cinco Expiatorio.

7. Motivo rítmico de seisillo en Chapala de Lobato, Juegos del agua de Ravel y Jardines bajo la lluvia de Debussy respectivamente. 